\title{
NON-LINEAR OPTIMIZATION OF THE MATERIAL CONSTANTS IN OGDEN'S STRESS-DEFORMATION FUNCTION FOR INCOMPRESSIBLE ISOTROPIC ELASTIC MATERIALS
}

\author{
E. H. TWIZELL and R. W. OGDEN'
}

(Received 4 November 1981; revised 30 March 1982)

\begin{abstract}
In previous papers, three terms have been included in Ogden's stress-deformation function for incompressible isotropic elastic materials. The material constants have been calculated by elementary methods and the resulting fits to sets of experimental data have been moderately good.

The purpose of the present paper is to improve upon established correlation between theory and experiment by means of a systematic optimization procedure for calculating material constants. For purposes of illustration the Levenberg-Marquardt non-linear least squares optimization algorithm is adapted to determine the material constants in Ogden's stress-deformation function.

The use of this algorithm for three-term stress-deformation functions improves somewhat on previous results. Calculations are also carried out in respect of a four-term stress-deformation function and further improvement in the fit is achieved over a large range of deformation.
\end{abstract}

\section{Introduction}

In a number of publications (see, for example, Ogden [6], Chadwick et al. [1]), elementary methods have been used to determine the material constants $\mu_{l}, \alpha_{t}$ in the stress-deformation function

$$
F \equiv \sum_{i=1}^{M} \mu_{i} p\left(\lambda, \alpha_{i}, c\right)=\sum_{i=1}^{M} \mu_{i}\left(\lambda^{-1+\alpha_{i}}-\lambda^{-1+c \alpha_{i}}\right)
$$

\footnotetext{
'Department of Mathematics, Brunel University, Uxbridge, Middlesex, England UB8 3PH.

(c) Copyright Australian Mathematical Society 1983
} 
for incompressible isotropic elastic materials. In equation (1), $F$ represents the force per unit undeformed area, normal to the principal direction of strain, corresponding to the principal stretch $\lambda$. The units of the $\mu_{i}$ are those of $F$ and the $\alpha_{1}$ are dimensionless $(i=1, \ldots, M)$. Considerations of stability and physically realistic response lead to the inequalities

$$
\mu_{t} \alpha_{\imath}>0 \text { for all } i=1, \ldots, M \text {. }
$$

The parameter $c$ in equation (1) is related to the pure homogeneous deformation of simple tension, pure shear and equibiaxial tension, for which $c=-\frac{1}{2},-1,-2$ respectively. For further details of the three deformations and the derivation of the corresponding values of $c$, the reader is referred to Ogden [6].

The set of numerical results for $\mu_{i}$ and $\alpha_{i}(i=1,2,3)$ given by Ogden [6] were obtained by an ad hoc method as were the two sets given by Chadwick et al. [1]; all of these values of the constants were derived to fit curves to the experimental data of Treloar [9]. Treloar's data were obtained in three experiments on samples cut from a single sheet of vulcanized natural rubber; his three sets of data are plotted for simple tension, pure shear and equibiaxial tension in Figures 1, 2, 3 respectively. A brief review of other experiments and associated fitted curves by Jones and Treloar [3], James et al. [2] and Treloar and Riding [10] is contained in Ogden [7].

Chadwick et al. [1] and Ogden [6] obtained values of $\mu_{i}$ and $\alpha_{t}(i=1,2,3)$ by using the fact that, at small strains $(\lambda \simeq 1)$, the computation is dominated by just one term $\mu_{1} p\left(\lambda, \alpha_{1}, c\right)$, with $\mu_{2} p\left(\lambda, \alpha_{2}, c\right)$ and $\mu_{3} p\left(\lambda, \alpha_{3}, c\right)$ increasing in importance as $\lambda$ increases. The actual values of $\mu_{t}, \alpha_{t}$ determined by Chadwick $e t$ al. [1] and Ogden [6] for the data of Treloar [9] are reproduced for comparison purposes in Table 1.

In previous papers, authors using the stress-deformation formula (1), have taken $M=3$. It was observed by Ogden [6, page 578], however, that by taking $M=4$, a better fit could be obtained for $\lambda>7.0$. One purpose of this paper is to report numerical results which verify this claim, though it will be seen that Ogden's estimate of $\alpha_{4} \simeq 10$ is too low for Treloar's data. The other purpose of this paper is to show that superior numerical results for $\mu_{t}, \alpha_{l}(i=1,2, \ldots, M)$ are obtained using non-linear least squares optimization techniques (Section 2). Such techniques obviate the need to calculate the $\mu_{i}, \alpha_{i}(i=1,2, \ldots, M)$ successively by fitting curves to expanding ranges of data. The optimal values $\mu_{i}^{*}, \alpha_{i}^{*}(i=$ $1,2, \ldots, M)$ are determined as the elements of a vector. For the data of Treloar [9] the optimal values with $M=3,4$ are reported in Section 3 and, for $M=4$, the curves generated by (1) are plotted in Figures 1, 2, 3. Comparison with the values of Ogden [6] and Chadwick et al. [1] is presented in Table 1 and comparison of the accuracy obtained using non-linear optimization methods with the accuracy attained by Ogden [6] and Chadwick et al [1] in Table 2. 
TABLE 1. Numerical values of $\mu_{i}\left(\mathrm{~kg} \mathrm{~cm}^{-2}\right), \alpha_{i}(i=1, \ldots, M ; M=3$ or 4$)$

\begin{tabular}{|l|c|c|c|c|c|c|c|c|}
\hline \multicolumn{1}{|c|}{ Method } & $\mu_{1}$ & $\alpha_{1}$ & $\mu_{2}$ & $\alpha_{2}$ & $\mu_{3}$ & $\alpha_{3}$ & $\mu_{4}$ & $\alpha_{4}$ \\
\hline $\begin{array}{l}\text { Twizell and } \\
\text { Ogden } \\
M=4, c=-\frac{1}{2}\end{array}$ & 6.27 & 1.23 & -0.054 & -1.99 & 0.036 & 4.44 & $0.80(-15)$ & 19.49 \\
\hline $\begin{array}{l}\text { Twizell and } \\
\text { Ogden } \\
M=4, c=-1\end{array}$ & 6.26 & $1.2 !$ & -0.067 & -1.99 & 0.059 & 4.43 & 0.94 i 5$)$ & 19.49 \\
\hline $\begin{array}{l}\text { Twizell and } \\
\text { Ogden } \\
M=4, c=-2\end{array}$ & 6.17 & 1.26 & -0.091 & -2.01 & 0.046 & 4.26 & $1.00(-14)$ & 19.49 \\
\hline $\begin{array}{l}\text { Twizell and } \\
\text { Ogden } \\
M=3, c=-\frac{1}{2}\end{array}$ & 2.22 & 2.26 & -0.448 & -2.01 & $0.39(-6)$ & 10.01 & - & - \\
\hline $\begin{array}{l}\text { Twizell and } \\
\text { Ogden } \\
M=3, c=-1\end{array}$ & 3.31 & 1.79 & -0.689 & -1.99 & $0.20(-5)$ & 9.88 & - & - \\
\hline $\begin{array}{l}\text { Twizell and } \\
\text { Ogden } \\
M=3, c=-2\end{array}$ & 5.39 & 1.46 & -0.530 & -2.02 & $0.19(-5)$ & 9.68 & - & - \\
\hline $\begin{array}{l}\text { Ogden (1972) } \\
\text { all } c\end{array}$ & 6.3 & 1.3 & -0.1 & -2.0 & 0.012 & 5.0 & - & - \\
\hline $\begin{array}{l}\text { Chadwick } \text { et al. } \\
\text { (1977) equation (5.4) } \\
\text { all } c\end{array}$ & 3.0 & 2.0 & -0.81 & -1.25 & $0.37(-4)$ & 7.82 & - & - \\
\hline $\begin{array}{l}\text { Chadwick } \text { et al. } \\
\text { (1977) equation (5.5) } \\
\text { all } c\end{array}$ & 3.24 & 2.0 & -0.1 & -2.0 & $0.62(-5)$ & 8.7 & - & - \\
\hline
\end{tabular}

Whereas the main aim of the present paper is to provide a systematic numerical procedure which ensures a close overall fit to Treloar's data, it is emphasized that this is different from the aims of Ogden [6] and Chadwick et al. [1]. In particular Chadwick et al. [1] were motivated by the desire to obtain modifications of the neo-Hookean $\left(\alpha_{1}=2\right)$ and Mooney-Rivlin $\left(\alpha_{1}=2, \alpha_{2}=-2\right)$ strain-energy functions, these being widely accepted as prototype models of the behaviour of rubberlike materials. Also, the above authors did not obtain separate fits to Treloar's [9] simple tension, pure shear and equibiaxial tension data sets, as is done in the present paper, but obtained values of $\mu_{i}, \alpha_{i}(i=1,2,3)$ appropriate to all three deformations simultaneously. 
TABLE 2. Sums of squares $(S)$

\begin{tabular}{|c|c|c|c|}
\hline & \multicolumn{3}{|c|}{$S$} \\
\cline { 2 - 4 } & $c=-\frac{1}{2}$ & $c=-1$ & $c=-2$ \\
\hline $\begin{array}{c}\text { Twizell and Ogden } \\
M=4, c=-\frac{1}{2}\end{array}$ & 6.30 & - & - \\
\hline $\begin{array}{c}\text { Twizell and Ogden } \\
M=4, c=-1\end{array}$ & - & 0.36 & - \\
\hline $\begin{array}{c}\text { Twizell and Ogden } \\
M=4, c=-2\end{array}$ & - & - & 1.92 \\
\hline $\begin{array}{c}\text { Twizell and Ogden } \\
M=3, c=-\frac{1}{2}\end{array}$ & 12.80 & - & - \\
\hline $\begin{array}{c}\text { Twizell and Ogden } \\
M=3, c=-1\end{array}$ & - & 0.63 & - \\
\hline $\begin{array}{c}\text { Twizell and Ogden } \\
M=3, c=-2\end{array}$ & - & - & 2.47 \\
\hline Ogden (1972) & 302.90 & 1.60 & 3.91 \\
\hline $\begin{array}{c}\text { Chadwick } \text { et al. } \\
(1977) \text { equation (5.4) }\end{array}$ & 20.40 & 2.83 & 10.32 \\
\hline $\begin{array}{c}\text { Chadwick } \text { et al. } \\
(1977) \text { equation (5.5) }\end{array}$ & 16.10 & 1.44 & 4.42 \\
\hline
\end{tabular}

\section{A non-linear least squares algorithm}

In this section the Levenberg-Marquardt iterative algorithm for calculating the optimal values $\mu_{i}^{*}, \alpha_{i}^{*}(i=1, \ldots, M)$ is outlined. The algorithm was published in 1963 by Marquardt and is similar to the method published in 1944 by Levenberg. In these two papers the $L_{2}$ norm is used in the minimization process; Shrager and Hill [8] discuss the implementation of the Levenberg-Marquardt algorithm in the $L_{1}$ and $L_{\infty}$ norms. The $L_{1}$ norm is particularly beneficial when the experimental data for $\lambda$ and $F$ contain one or more wild points, and the $L_{\infty}$ norm when the errors in the experimental values of $F$ are negligible. The $L_{2}$ norm has enjoyed much more use than the other two, as error estimates have long been available in the literature. Consequently a large literature of successful applications of the Levenberg-Marquardt algorithm in the $L_{2}$ norm, and a large amount of associated computer software, has resulted. The Levenberg-Marquardt algorithm in the $L_{2}$ norm is available to IBM users as Share Problem \# 1428 and from the NAG (Numerical Algorithms Group) subroutine library where it is implemented in Fortran as E04GAF and in Algol as E04GAA. 
The data of Treloar [9] used in this paper contain no wild points and so the outline of the Levenberg-Marquardt algorithm which follows is related to the $L_{2}$ norm.

Suppose there are $K$ data pairs $\left(\lambda_{k}, F_{k}\right)$ for $k=1, \ldots, K$ with $K \geqslant 2 M$. Let $\hat{F}_{k}$ be the value of $F_{k}$ yielded by

$$
F_{k}=\sum_{i=1}^{M} \mu_{i}\left(\lambda_{k}^{-1+\alpha_{t}}-\lambda_{k}^{-1+c \alpha_{t}}\right)
$$

and let $E_{k}=F_{k}-\hat{F}_{k}$ be the error in $F_{k}$. The least squares criterion requires that

$$
\begin{aligned}
S & =S\left(\mu_{1}, \alpha_{1}, \ldots, \mu_{M}, \alpha_{M}\right)=\sum_{k=1}^{K} E_{k}^{2} \\
& =\sum_{k=1}^{K}\left\{F_{k}-\sum_{i=1}^{M} \mu_{i}\left(\lambda_{k}^{-1+\alpha_{i}}-\lambda_{k}^{-1+c \alpha_{i}}\right)\right\}^{2}
\end{aligned}
$$

be minimized; this minimum of $S$ being reached by obtaining optimal values $\mu_{i}^{*}, \alpha_{\imath}^{*}$ of the parameters $\mu_{\imath}, \alpha_{\imath}(i=1, \ldots, M)$. In order to implement the Levenberg-Marquardt algorithm to minimize $S$, it is convenient to introduce a vector $\mathbf{x}$ of order $2 M$ defined by

$$
\mathbf{x}=\left(x_{1}, x_{2}, \ldots, x_{2 M-1}, x_{2 M}\right)^{T}=\left(\mu_{1}, \alpha_{1}, \ldots, \mu_{M}, \alpha_{M}\right)^{T},
$$

where $T$ denotes transpose.

The Levenberg-Marquardt algorithm calculates iteratively a sequence of points $\mathbf{x}^{(r)}(r=0,1,2, \ldots)$ with $\mathbf{x}^{(0)}$ some initial point chosen so that the sequence $\left\{\mathbf{x}^{(r)}\right\}$ will converge to a point $\mathrm{x}^{*}=\left(\mu_{1}^{*}, \alpha_{1}^{*}, \ldots, \mu_{M}^{*}, \alpha_{M}^{*}\right)^{T}$ that minimizes $S$ (the superscript $r$ denoting the $r$ th iterate). The algorithm calculates the vector $\mathbf{x}^{(r+1)}$ from the vector $\mathbf{x}^{(r)}$ using the equation

$$
\mathbf{x}^{(r+1)}=\mathbf{x}^{(r)}-\left[\left(P^{(r)}\right)^{T} P^{(r)}+\gamma^{(r)} I\right]^{-1}\left(P^{(r)}\right)^{T} \mathbf{E}^{(r)}, \quad r=0,1,2, \ldots,
$$

where $\gamma^{(r)}(r=0,1,2, \ldots)$ is an arbitrary parameter and $\mathbf{E}=\left(E_{1}, E_{2}, \ldots, E_{k}\right)^{T}$ is the vector of errors (see equation (3)). The matrix $I$ is the identity matrix of order $2 M$ and $P$ is the matrix of first derivatives of order $K \times 2 M$ whose element $p_{k l}$ at the $r$ th iterate is given by

$$
p_{k i}^{(r)}=\left.\frac{\partial E_{k}}{\partial x_{\imath}}\right|_{\mathbf{x}=\mathbf{x}^{(r)}}, \quad(k=1, \ldots, K ; i=1, \ldots, 2 M ; r=0,1,2, \ldots) .
$$

Thus

$$
p_{k, 2 l-1}^{(r)}=-\left\{\lambda_{k}^{-1+\alpha(r)}-\lambda_{k}^{-1+c \alpha \gamma^{(r)}}\right\}
$$


and

$$
p_{k, 2 l}^{(r)}=-\mu_{l}^{(r)}\left\{\lambda^{-1+\alpha \gamma^{(r)}}-c \lambda_{k}^{-1+c \alpha \gamma^{\prime \prime}}\right\} \ln \lambda_{k}
$$

for $k=1, \ldots, K ; l=1, \ldots, M ; r=0,1,2, \ldots$.

Marquardt [5] has shown that a sufficiently large $\gamma^{(r)}$ always exists such that

$$
S^{(r+1)}<S^{(r)}
$$

(unless $\mathbf{x}^{(r)}=\mathbf{x}^{*}$ ), where $S^{(r)}$ denotes the value of $S$ at the $r$ th iteration ( $r=$ $0,1,2, \ldots)$. It is clear therefore that the method converges from poor starting values $\mu_{i}^{(0)}, \alpha_{i}^{(0)}(i=1, \ldots, M)$ and convergence proceeds as follows:

(i) arbitrarily choose $\gamma^{(0)}$ and a parameter $u>1$; say $\gamma^{(0)}=0.01$ and $u=10$;

(ii) let $T\left(\gamma^{(r)}\right), T\left(\gamma^{(r)} / u\right)$ be the values of $S^{(r)}$ when $\gamma^{(r)}$ and $\gamma^{(r)} / u$, respectively, are used in equation (3);

(iii) calculate $S^{(r+1)}, T\left(\gamma^{(r)}\right)$ and $T\left(\gamma^{(r)} / u\right)$;

(iv) then

(a) if $T\left(\gamma^{(r)} / u\right) \leqslant S^{(r+1)}$, let $\gamma^{(r+1)}=\gamma^{(r)} / u$;

(b) if $T\left(\gamma^{(r)} / u\right)>S^{(r+1)}$ and $T\left(\gamma^{(r)}\right) \leqslant S^{(r+1)}$, let $\gamma^{(r+1)}=\gamma^{(r)}$;

(c) if $T\left(\gamma^{(r)} / u\right)>S^{(r+1)}$ and $T\left(\gamma^{(r)}\right)>S^{(r+1)}$, increase $\gamma^{(r)}$ by successive multiplication by $u$ until the positive integer $n$ is reached such that $T\left(\gamma^{(r)} u^{n}\right) \leqslant$ $S^{(r+1)}$. Let $\gamma^{(r+1)}=\gamma^{(r)} / u^{n}$

(v) test for convergence of all the material constants $\mu_{l}, \alpha_{l}(i=1, \ldots, M)$ to the required accuracy. If the accuracy criterion is met the iterations cease, otherwise $r$ is incremented by unity and control returns to (ii).

The convergence tests described in steps (iv) and (v) of the strategy do lead to increased computer time and storage in comparison with less sophisticated methods. With $\gamma^{(r)} \equiv 0$, for instance, the Levenberg-Marquardt algorithm (4) becomes the Gauss-Newton algorithm which, for some problems, may well converge faster, from good initial values, than the Levenberg-Marquardt algorithm. From poor initial values, however, the Gauss-Newton method may diverge while the Levenberg-Marquardt algorithm will converge. It is this factor which highlights the superior reliability of the Levenberg-Marquardt algorithm, and which makes it especially valuable for fitting a curve of the form (1) to experimental data.

\section{Numerical results}

The optimal values of $\mu_{\imath}, \alpha_{i}(i=1,2,3,4)$ were computed using the Levenberg-Marquardt algorithm for the laboratory data of Treloar [9] relating to his simple tension, pure shear and equibiaxial tension experiments for which 
$c=-\frac{1}{2},-1,-2$ respectively. These optimal values for $M=4$ are contained in Table 1 . With the exception of $\alpha_{4}$, corresponding material constants vary slightly between experiments. Ideally, this variation would not happen and a possible explanation for its occurrence is the presence of errors in experimental observations. The minimum sums of squares, defined by equation (3), were then computed for the three sets of material constants with their corresponding sets of experimental data; these three values of $S$ are contained in Table 2.

The two sets of material constants obtained by Ogden [6] and Chadwick $e t$ al. [1] all with $M=3$, are contained in Table 1 and the resulting values of $S$ are contained in Table 2. The values of $S$ obtained in the present paper for $M=4$ using the non-linear least squares algorithm, are seen to be smaller, leading to a better overall fit to each of the three sets of experimental data.

Sets of optimal values of $\mu_{i}, \alpha_{i}(i=1,2,3)$ were also computed using the Levenberg-Marquardt algorithm for the same laboratory data of Treloar [9]. These optimal values for $M=3$ are also contained in Table 1 and the corresponding values of $S$ are contained in Table 2. The three values of $S$ obtained using the material constants determined for $M=3$ are again seen to be smaller than those obtained by Chadwick et al. [1] and Ogden [6]. They are not, however, as small as those obtained with $M=4$.

It is seen that for $M=3$ the values of $\mu_{1}, \alpha_{1}, \mu_{2}, \mu_{3}$ for different tests ( $\left.c=-\frac{1}{2},-1,-2\right)$ differ considerably, but for $M=4$ the maximum difference between tests is very small. This justifies the introduction of the fourth term, although there is no obvious physical basis for it. The differences in values of the material constants for $M=4$ are small enough not to be detectable within bounds of experimental error, and the set of values

$$
\begin{gathered}
\mu_{1}=6.23, \mu_{2}=-0.071, \mu_{3}=0.047, \mu_{4}=0.91 \times 10^{-15}\left(\mathrm{~kg} \mathrm{~cm}^{-2}\right) \\
\alpha_{1}=1.23, \alpha_{2}=-1.99, \alpha_{3}=4.38, \alpha_{4}=19.49
\end{gathered}
$$

may be taken to cover all three tests.

In Figures 1, 2, 3 the curves for simple tension, pure shear and equibiaxial tension are plotted for $M=4$ and compared with the data points of Treloar [9]. Also included for comparison are the corresponding curves generated by the material constants of Ogden [6] and Chadwick et al. [1, page 74]. The figures confirm that the use of four terms in the stress-deformation function (1) gives a very close fit overall for all three experiments. The comparison with Ogden's results should be qualified with the fact that he omitted one of Treloar's simple tension data points in calculating his material constants (two from his Figure 1).

The sets of optimal values of $\mu_{t}, \alpha_{i}(i=1, \ldots, M)$ computed for both $M=3$ and $M=4$ by the Levenberg-Marquardt algorithm clearly satisfy the inequalities 


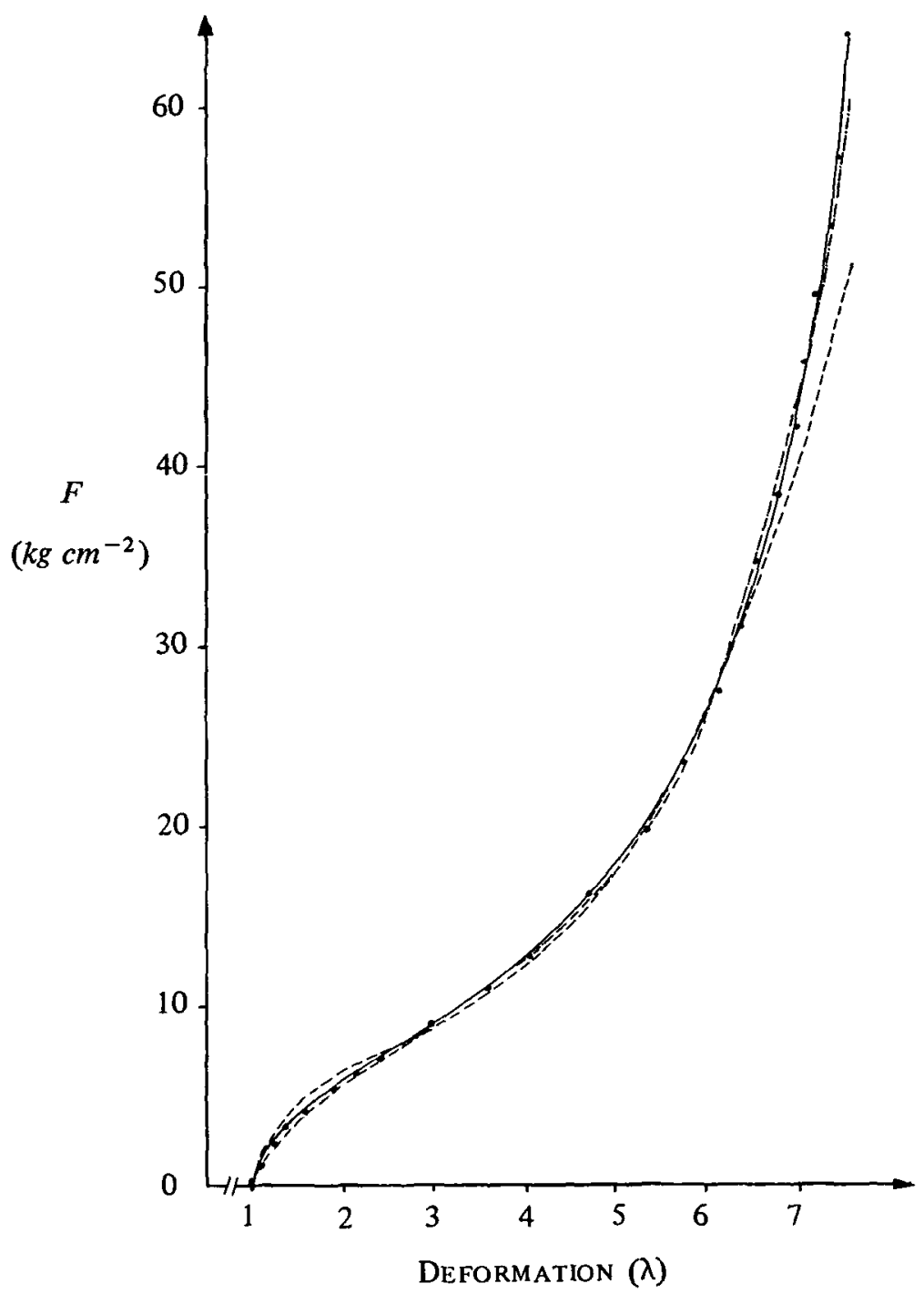

Figure 1. Curves for Treloar's simple tension data.

Twizell and Ogden, $M=4$;

Ogden [6];

Chadwick et al. [1]

$\ldots \ldots$. Treloar's data.

(2). However, of the optimal values $\alpha_{1}^{*}$ for $M=3$ only that corresponding to $c=-\frac{1}{2}$ satisfies the condition

$$
\alpha_{i} \leqslant-1 \text { or } \alpha_{i} \geqslant 2
$$




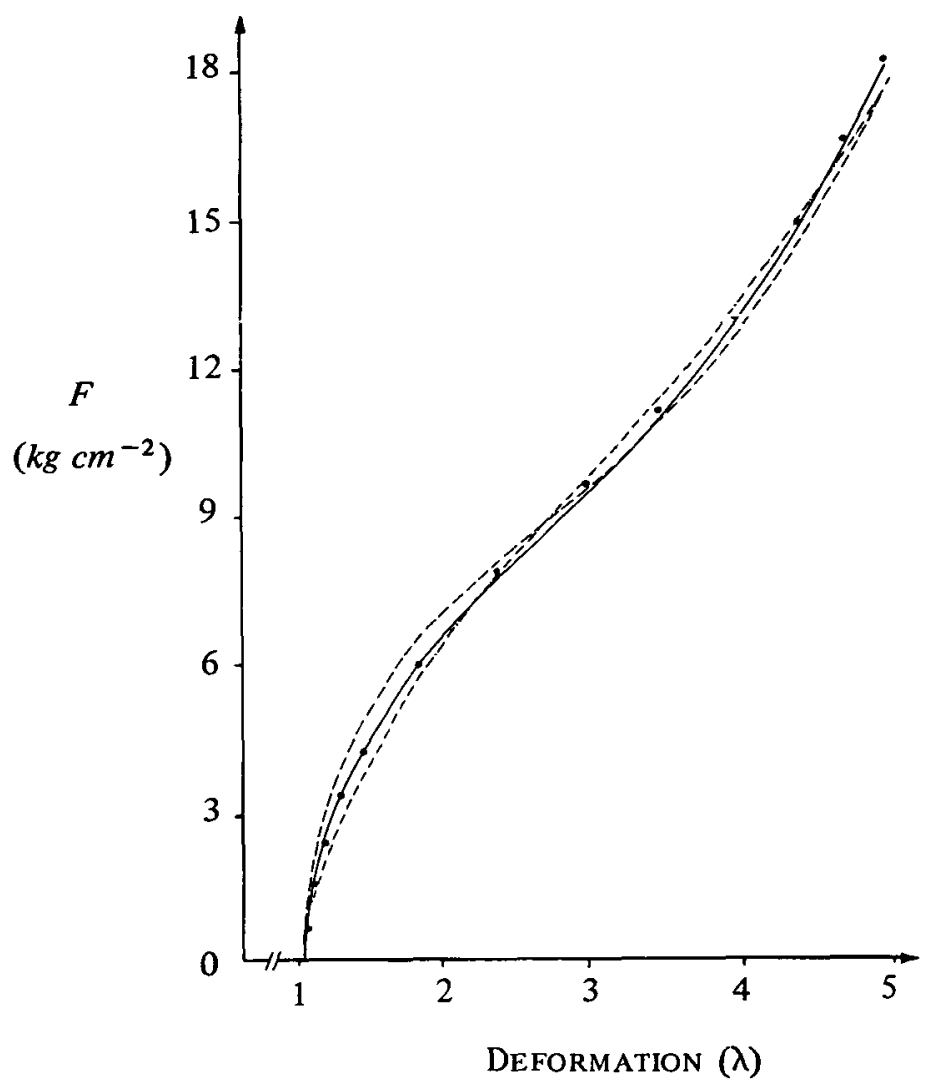

Figure 2. Curves for Treloar's pure shear data.

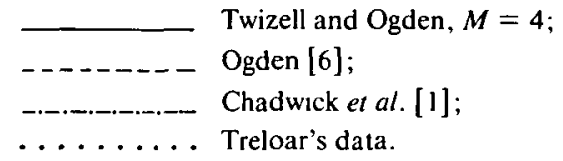

(Chadwick et al. [1, page 63]). These conditions are sufficient to guarantee the existence of a unique solution to the problem of a shape-preserving deformation of a circular cylinder rotating about its axis. In their paper, Chadwick et al. [1] reject Ogden's value of $\alpha_{1}=1.3$ in [6] because it, too, violates (7); it is noted that for all three values of $c$ with $M=4$, the Levenberg-Marquardt algorithm yields optimal values $\mu_{1}^{*}$ and $\alpha_{1}^{*}$ which are very close to those of Ogden [6]. The values of $\alpha_{1}^{*}$ computed using the non-linear least squares method with $M=3$ and $c=-1$, -2 also violate (7), yet the corresponding values of $S$ are smaller than those 


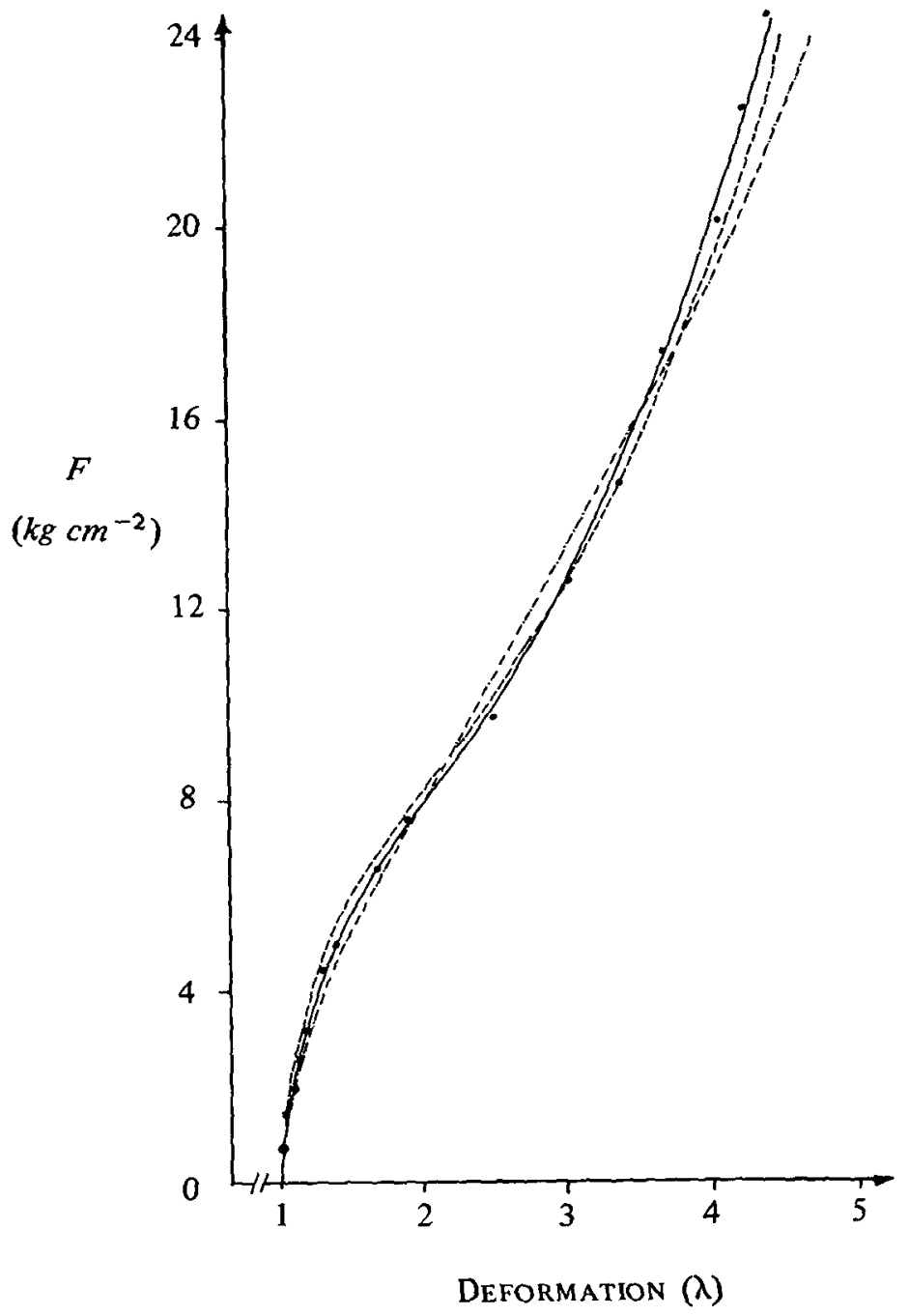

Figure 3. Curves for Treloar's equibiaxial tension data.

Twizell and Ogden, $M=4$;

_......... Ogden [6];

............... Chadwick et al. [1];

....... Treloar's data.

obtained by either Chadwick et al. [1] or Ogden [6]. Grounds for accepting values of $\alpha_{t}$ which violate (7) are discussed in Ogden [7]. Indeed such values of $\alpha_{t}$ are necessary, for example, for bifurcation from a spherical configuration of an inflated, initially spherical shell. 


\section{Summary}

The numerical results reported in the present paper verify that the use of non-linear least squares optimization methods is justified when fitting curves of the form (1) to the experimental data; the wide availability of relevant computer software enforces this point.

It has further been verified that the use of four terms in Ogden's stress-deformation function produces a cioser overall fit than the use of three terms, as might be expected. Moreover, the values of the $\mu_{i}, \alpha_{i}(i=1, \ldots, M)$ for different tests are much closer for $M=4$ than $M=3$, and provide a single set of material constants for all the three tests within the bounds of experimental error.

\section{Acknowledgements}

The authors acknowledge the financial support of the Science and Engineering Research Council to attend the Strathclyde Biomechanics Workshop in July 1980 at the University of Strathclyde, Glasgow, Scotland, where the research for this paper began. The authors are grateful for the constructive criticisms of an anonymous referee and of Dr. V. G. Hart, Department of Mathematics, University of Queensland, which led to improvements in the paper.

\section{References}

[1] P. Chadwick, C. F. M. Creasy and V. G. Hart, "The deformation of rubber cylinders and tubes by rotation", J. Austral. Math. Soc. Ser. B 20 (1977), 62-96.

[2] A. G. James, A. Green and G. M. Simpson, "Strain-energy functions of rubber I: Characterization of gum vulcanizates", J. Appl. Polymer Scl. 19 (1975), 2033-2058.

[3] D. F. Jones and L. R. G. Treloar, "The properties of rubber in pure homogeneous strain", $J$. Phys. D: Appl. Phys. 8 (1975), 1285-1304.

[4] K. Levenberg, "A method for the solution of certain non-linear problems in least squares", Quart. Appl. Math. 2 (1944), 164-168.

[5] D. W. Marquardt, “An algorithm for least squares estimation of non-linear parameters", SIAM J. Appl. Math. 11 (1963), 431-441.

[6] R. W. Ogden, "Large deformation isotropic elasticity-on the correlation of theory and experiment for incompressible rubberlike solids", Proc. Roy. Soc. London Ser. A 326 (1972), 565-584.

[7] R. W. Ogden, "Elastic deformations of rubberlike solids", in Mechanics of Solids, The Rodney Hill 60th Anniversary Volume (eds. H. G. Hopkins and M. J. Sewell), (Pergamon Press. Oxford, 1982).

[8] R. I. Shrager and E. Hill Jr., "Non-linear curve fitting in the $L_{1}$ and $L_{\infty}$ norms", Math. Comp. 34 (1980), 529-541.

[9] L. R. G. Treloar, "Stress-strain data for vulcanised rubber under various types of deformation", Trans. Faraday Soc. 40 (1944), 59-70.

[10] L. R. G. Treloar and G. Riding, "A non-Gaussian thory for rubber in biaxial strain I: Mechanical properties", Proc. Roy. Soc. London Ser. A 369 (1980), 261-280. 